\title{
THE VITALI-HAHN-SAKS THEOREM AND MEASUROIDS
}

\author{
SADAHIRO SAEKI
}

(Communicated by Andrew M. Bruckner)

\begin{abstract}
We introduce the concept of measuroids, and prove the Uniform Boundedness Principle of Nikodym-Grothendieck, the Vitali-Hahn-Saks theorem and the Nikodym Convergence Theorem for measuroids.
\end{abstract}

The theorem in the title asserts that the setwise limit of a sequence of probability measures is again a probability measure. The present note gives some versions of this classical result and of some related results. Most of the ideas in our proofs are quite standard and can be found in the beautiful treatment [3] by Diestel; see also the lecture notes [1] by Antosik-Swartz.

In the sequel, let $(\Omega, \mathscr{M})$ denote a fixed measurable space. We begin with a proof of the following "primitive" result.

Theorem 1. Let $\left(\mu_{n}\right)_{1}^{\infty}$ be a sequence of bounded positive measures on $\mathscr{M}$, and let $\psi: \mathscr{M} \rightarrow[0, \infty]$ be defined by

$$
\psi(A)=\limsup _{n \rightarrow \infty} \mu_{n}(A) .
$$

Then we have either

(a) $\psi\left(A_{n}\right) \rightarrow 0$ for each sequence $\left(A_{n}\right)_{1}^{\infty}$ in $\mathscr{M}$ with $A_{n} \downarrow \varnothing$, or

(b) $\inf _{n} \psi\left(B_{n}\right)>0$ for some disjoint sequence $\left(B_{n}\right)_{1}^{\infty}$ in $\mathscr{M}$.

Proof. Suppose that (a) is not the case. Then there exists $\beta>0$ and $A_{p} \in$ $\mathscr{M}(p \in \mathbb{N})$ such that $A_{p} \downarrow \varnothing$ and

$$
\psi\left(A_{p}\right)>\beta \quad \forall p \geq 1 .
$$

We choose natural numbers $n_{1}<p_{1}<n_{2}<p_{2}<\cdots$ as follows. Let $n_{0}=p_{0}=$ 1 and suppose that $n_{0}, p_{0}, \ldots, n_{k-1}, p_{k-1}$ have been defined. By $(*)$ and (1), there exists $n_{k}>p_{k-1}$ such that $\mu_{n_{k}}\left(A_{p_{k-1}}\right)>\beta$. Moreover, each $\mu_{n}$ is a bounded measure and $A_{p} \downarrow \varnothing$. Hence $\mu_{n_{k}}\left(A_{p}\right) \rightarrow 0$ as $p \rightarrow \infty$. So we can find $p_{k}>n_{k}$ such that

Received by the editors October 3, 1990.

1980 Mathematics Subject Classification (1985 Revision). Primary 28A33, 28B05; Secondary $46 \mathrm{G} 10$.

Key words and phrases. Vitali-Hahn-Saks theorem, Uniform Boundedness Principle, Nikodym Convergence Theorem. 


$$
\mu_{n_{k}}\left(A_{p_{k-1}} \backslash A_{p_{k}}\right)>\beta,
$$

which completes our induction.

Let $D_{k}=A_{p_{k-1}} \backslash A_{p_{k}}$ for $k \geq 1$, and let $B_{I}=\bigcup_{k \in I} D_{k}$ for each infinite set $I \subset \mathbb{N}$. Then $k \in I$ implies $\mu_{n_{k}}\left(B_{I}\right) \geq \mu_{n_{k}}\left(D_{k}\right)>\beta$ by (2). Therefore

$$
\psi\left(B_{I}\right) \geq \limsup _{k \rightarrow \infty} \mu_{n_{k}}\left(B_{I}\right) \geq \beta \text {. }
$$

Moreover, $I \cap J=\varnothing$ implies $B_{I} \cap B_{J}=\varnothing$. So, to complete the proof, it suffices to write $\mathbb{N}$ as an infinite disjoint of infinite sets.

Now we introduce the concept of measuroids. A measuroid on $\mathscr{M}$ is a function $\phi: \mathscr{M} \rightarrow[0, \infty]$, with $\phi(\varnothing)=0$, such that $A, B \in \mathscr{M}$ and $A \cap B=\varnothing$ implies

$$
\begin{gathered}
\phi(A \cup B) \leq \phi(A)+\phi(B), \\
\phi(A) \leq \phi(A \cup B)+\phi(B) .
\end{gathered}
$$

Here (SA) and (SB) stand for "subadditive" and "subbalanced," respectively. A measuroid $\phi$ is said to be bounded iff $\sup _{A} \phi(A)<\infty$; continuous (at $\varnothing$ ) iff $A_{n} \downarrow \varnothing$ in $\mathscr{M}$ implies $\phi\left(A_{n}\right) \rightarrow 0$; and vanishing iff $\phi\left(B_{n}\right) \rightarrow 0$ for each disjoint sequence $\left(B_{n}\right)_{1}^{\infty}$ in $\mathscr{M}$. Note that every continuous measuroid is vanishing; simply take $A=B_{n}$ and $B=\bigcup_{k>n} B_{k}$ in (SB). Then function $\psi$ in Theorem 1 is obviously a measuroid, and the conclusion of Theorem 1 says that $\psi$ is either continuous or else nonvanishing.

Examples. (a) Let $X$ be a Banach space, let $\mu: \mathscr{M} \rightarrow X$ be finitely additive, and let

$$
\mu^{\#}(A)=\sup \{\|\mu(B)\|: B \in \mathscr{M} \text { and } B \subset A\}
$$

for $A \in \mathscr{M}$. Then both $A \rightarrow\|\mu(A)\|$ and $A \rightarrow \mu^{\#}(A)$ are measuroids. Note that $\mu$ is countably (resp. strongly) additive if and only if $A \rightarrow\|\mu(A)\|$ is continuous (resp. vanishing). An easy application of a result of Bessaga and Pelczynski [2] shows that if $X$ contains no copy of $c_{0}$ as a closed subspace and, if $\mu$ is bounded (i.e., $\left.\mu^{\#}(\Omega)<\infty\right)$, then $\mu$ is strongly additive. By a result of Diestel and Faires [4], this result holds with $c_{0}$ replaced by $\ell_{\infty}$.

(b) Let $\mu$ be a positive measure on $\mathscr{M}$, let $0<p \leq \infty$, and let $T$ be a mapping from $L_{p}(\mu)$ into the space of all nonnegative measurable functions on $\Omega$ such that

$$
T 0=0, \quad T(f+g) \leq T f+T g \text { and } T(-f)=T f
$$

for $f, g \in L_{p}(\mu)$. Fix any $f \in L_{p}(\mu)$, and define $\phi(A)=\int T\left(\chi_{A} f\right) d \mu$ for each $A \in \mathscr{M}$, where $\chi_{A}$ is the characteristic function of $A$. Then $\phi$ is a measuroid.

(c) Let $\phi$ be a measuroid, and let

$$
\phi^{\#}(A)=\sup \{\phi(B): B \in \mathscr{M} \text { and } B \subset A\}
$$

for each $A \in \mathscr{M}$. Then $\phi^{\#}$ is a measuroid on $\mathscr{M}$.

Lemma 2. Let $\phi$ be a measuroid on $\mathscr{M}$.

(a) Suppose $\phi$ is vanishing, $\left(B_{n}\right)_{1}^{\infty}$ is a disjoint sequence in $\mathscr{M}$, and $\varepsilon>0$. Then $\phi^{\#}$ is vanishing and $\mathbb{N}$ contains an infinite set $I$ such that $\phi^{\#}\left(\bigcup_{k \in I} B_{k}\right)$ $<\varepsilon$. 
(b) If $\phi$ is continuous, then $\phi^{\#}$ is also continuous.

Proof. (a) Write $\mathbb{N}$ as the infinite disjoint union of infinite sets $I_{n} \subset \mathbb{N}$, and let $U_{n}=\bigcup\left\{B_{k}: k \in I_{n}\right\}$ for $n \geq 1$. Choose measurable set $E_{n} \subset U_{n}$ so that $\phi\left(E_{n}\right)>\min \left\{\phi^{\#}\left(U_{n}\right), n\right\}-1 / n$. Since the $E_{n}$ 's are pairwise disjoint, it follows that $\phi\left(E_{n}\right) \rightarrow 0$; hence $\phi^{\#}\left(U_{n}\right) \rightarrow 0$. To see that $\phi^{\#}$ is vanishing, apply the above argument to $\left(B_{k}\right)_{1}^{\infty}$ in place of $\left(U_{n}\right)_{1}^{\infty}$.

(b) Suppose $\phi$ is continuous but $\phi^{\#}$ is not. Then there exists $A_{p} \in \mathscr{M}$ and $\beta>0$ such that $A_{p} \downarrow \varnothing$ and $\phi^{*}\left(A_{p}\right)>\beta$ for every $p \geq 1$. Let $D_{0}=\varnothing$ and $p_{0}=1$. Suppose that $D_{0}, \ldots, D_{k-1} \in \mathscr{M}$ and $p_{0}, \ldots, p_{k-1} \in \mathbb{N}$ have been given for some $k \geq 1$. Choose any measurable sets $B_{k} \subset A_{p_{k-1}}$ with $\phi\left(B_{k}\right)>\beta$. Note that

$$
\begin{aligned}
\phi\left(B_{k}\right) & \leq \phi\left(B_{k} \backslash A_{p}\right)+\phi\left(B_{k} \cap A_{p}\right) \quad \text { by (SA) } \\
& =\phi\left(B_{k} \backslash A_{p}\right)+o(1) \quad \text { as } p \rightarrow \infty \text { by Continuity }
\end{aligned}
$$

since $A_{p} \downarrow \varnothing$. So we can find $p_{k}>p_{k-1}$ such that

$$
\phi\left(D_{k}\right)>\beta,
$$

where $D_{k}=B_{k} \backslash A_{p_{k}} \subset A_{p_{k-1}} \backslash A_{p_{k}}$. Continuing this process, we obtain a disjoint sequence $\left(D_{k}\right)_{1}^{\infty}$ in $\mathscr{M}$ which satisfies (3) for each $k \geq 1$. Plainly this contradicts the vanishing property of $\phi$, which establishes $(b)$.

Lemma 3. Let $\left(\phi_{n}\right)_{1}^{\infty}$ be a sequence of vanishing measuroids, and let $\left(B_{n}\right)_{1}^{\infty}$ be a disjoint sequence in $\mathscr{M}$. Then there exists $I \subset \mathbb{N}$ such that $\phi_{n}\left(B_{I}\right) \geq \phi_{n}\left(B_{n}\right) / 2$ for infinitely many $n$ 's, where $B_{I}=\bigcup_{j \in I} B_{j}$.

Proof. We may suppose that for each finite set $F \subset \mathbb{N}, \phi_{n}\left(B_{F}\right)<\phi_{n}\left(B_{n}\right) / 2$ for all $n$ 's large enough (otherwise, take $I=F$ ). We choose infinite sets $I_{1}, I_{2}, \ldots$ in $\mathbb{N}$ and natural numbers $n_{1}, n_{2}, \ldots$ as follows. Let $I_{1}=\mathbb{N}$, and pick any $n_{1} \in \mathbb{N}$ with $\phi_{n_{1}}\left(B_{n_{1}}\right) \neq 0$. Let $k \geq 1$ be given, and suppose that $I_{1}, \ldots, I_{k}, n_{1}, \ldots, n_{k}$ have been chosen in such a way that

$$
\phi_{n_{k}}\left(\bigcup_{j=1}^{k-1} B_{n_{j}}\right)<\phi_{n_{k}}\left(B_{n_{k}}\right) / 2 \text {. }
$$

By the hypotheses, $\phi_{n_{k}}$ is vanishing and the $B_{n}$ 's are pairwise disjoint. So Lemma 2(a) and (4) ensure that $I_{k}$ contains an infinite set $I_{k+1}$ such that

$$
\phi_{n_{k}}^{\#}\left(\bigcup_{j \in I_{k+1}} B_{j}\right)<\phi_{n_{k}}\left(B_{n_{k}}\right) / 2-\phi_{n_{k}}\left(\bigcup_{j=1}^{k-1} B_{n_{j}}\right) \text {. }
$$

By our additional assumption, we can find $n_{k+1} \in I_{k+1}$, with $n_{k+1}>n_{k}$, so that (4) holds with $k$ replaced by $k+1$, which completes the induction. Hence

Let $I=\left\{n_{k}\right\}_{1}^{\infty}$. By the above construction, $k \geq 1$ implies $\left\{n_{j}\right\}_{j=k+1}^{\infty} \subset I_{k+1}$.

$$
\begin{aligned}
\phi_{n_{k}}\left(B_{I} \backslash B_{n_{k}}\right) & \leq \phi_{n_{k}}\left(\bigcup_{j=1}^{k-1} B_{n_{j}}\right)+\phi_{n_{k}}\left(\bigcup_{j=k+1}^{\infty} B_{n_{j}}\right) \quad \text { by (SA) } \\
& <\phi_{n_{k}}\left(B_{n_{k}}\right) / 2 \text { by (5). }
\end{aligned}
$$


If $\phi_{n_{k}}\left(B_{I}\right)<\infty$, it follows that

$$
\begin{aligned}
\phi_{n_{k}}\left(B_{n_{k}}\right) & \leq \phi_{n_{k}}\left(B_{I} \backslash B_{I_{n_{k}}}\right)+\phi_{n_{k}}\left(B_{I}\right) \quad \text { by }(\mathrm{SB}) \\
& <\phi_{n_{k}}\left(B_{n_{k}}\right) / 2+\phi_{n_{k}}\left(B_{I}\right) \text { by }(6) .
\end{aligned}
$$

Hence $\phi_{n_{k}}\left(B_{I}\right) \geq \phi_{n_{k}}\left(B_{n_{k}}\right) / 2$ in either case, which completes the proof.

Now we are ready to prove the Uniform Boundedness Principle of NikodymGrothendieck for vanishing measuroids.

Theorem 4. Let $S$ be a set of vanishing measuroids such that

$$
\psi(A):=\sup \{\phi(A): \phi \in S\}<\infty
$$

for each $A \in \mathscr{M}$. Then $\sup \left\{\phi^{\#}(\Omega): \phi \in S\right\}<\infty$.

Proof. Plainly $\psi$ is a measuroid. We need to show that $\psi^{\#}(\Omega)<\infty$.

Suppose to the contrary that $\psi^{\#}(\Omega)=\infty$. Pick any $A \in \mathscr{M}$ with $\psi^{\#}(A)=\infty$ (e.g., $A=\Omega$ ). Then we can find $B \subset A$ and $\phi \in S$ such that $\phi(B)$ is arbitrarily large. Note that

$$
\psi^{\#}(B)+\psi^{\#}(A \backslash B) \geq \psi^{\#}(A)=\infty \quad \text { by }(\mathrm{SA}),
$$

so $\psi^{\#}(B)=\infty$ or $\psi^{\#}(A \backslash B)=\infty$. Moreover, we have

$$
\begin{aligned}
\phi(B) & \leq \phi(A)+\phi(A \backslash B) \quad \text { by }(\mathrm{SB}) \\
& \leq \psi(A)+\phi(A \backslash B) \quad \text { by }(*) .
\end{aligned}
$$

Thus $\phi(A \backslash B) \geq \phi(B)-\psi(A)$, which is arbitrarily large. So upon interchanging $B$ and $A \backslash B$ if necessary, we may suppose that $\phi(B)$ is large enough and $\psi^{\#}(A \backslash B)=\infty$.

An induction application of the above observation yields a disjoint sequence $\left(B_{n}\right)_{1}^{\infty}$ in $\mathscr{M}$ and a sequence $\left(\phi_{n}\right)_{1}^{\infty}$ in $S$ such that $\phi_{n}\left(B_{n}\right)>n$ for every $n \geq 1$. Therefore Lemma 3 ensures the existence of $B_{I} \in \mathscr{M}$ such that $\sup _{n} \phi_{n}\left(B_{I}\right)=\infty$, which contradicts $(*)$.

Now let $\mu$ and $\phi$ be two measuroids. We say that $\phi$ is $\mu$-a.c. (absolutely continuous) if and only if given $\varepsilon>0$, there exists $\delta>0$ such that $A \in \mathscr{M}$ and $\mu^{\#}(A)<\delta$ implies $\phi(A)<\varepsilon$. If $S$ is a set of measuroids and $\psi_{S}(A)=$ $\sup \left\{\phi^{\#}(A): \phi \in S\right\}$ for each $A \in \mathscr{M}$, then $\psi_{S}$ is a measuroid. $S$ is said to be equi-vanishing, equi-continuous, or equi- $\mu$-a.c. iff $\psi_{S}$ is vanishing, continuous, or $\mu$-a.c., respectively. For a vector measure $\nu$ and a collection of such, we define the above concepts with $\phi$ replaced by $A \rightarrow\|\nu(A)\|$.

Lemma 5. Let $\mu$ be a countably subadditive measuroid, let $\left(\phi_{n}\right)_{1}^{\infty}$ be a sequence of $\mu$-a.c. measuroids, and let $\left(B_{n}\right)_{1}^{\infty}$ be a disjoint sequence in $\mathscr{M}$ with $\mu^{\#}\left(B_{n}\right) \rightarrow$ 0 . Then there exists $I \subset \mathbb{N}$ such that $\phi_{n}\left(B_{I}\right) \geq \phi_{n}\left(B_{n}\right) / 2$ for infinitely many $n$ 's, where $B_{I}=\bigcup_{j \in I} B_{j}$.

Proof. As $\mu$ is countably subadditive, it is easily seen that $\mu^{\#}$ is also countably subadditive. So, passing to a subsequence, we may suppose that $\mu^{\#}\left(\bigcup_{j=n}^{\infty} B_{j}\right) \rightarrow$ 0 as $n \rightarrow \infty$. Then the proof of the present lemma is exactly the same as the proof of Lemma 3 , except that the existence of an infinite set $I_{k+1} \subset I_{k}$ satisfying (5) is now guaranteed by the $\mu$-absolute continuity of $\phi_{n_{k}}$. 
Theorem 6. Let $\mu$ be a countably subadditive measuroid, let $\left(\phi_{n}\right)_{1}^{\infty}$ be a sequence of $\mu$-a.c. (resp. vanishing, or continuous) measuroids, and let

$$
\psi(A)=\limsup _{n \rightarrow \infty} \phi_{n}(A)
$$

for each $A \in \mathscr{M}$. If $\psi$ is vanishing, then the $\phi_{n}$ 's are equi- $\mu$-a.c. (resp. equivanishing, or equi-continuous); hence $\psi$ is $\mu$-a.c. (resp. vanishing, or continuous).

Proof. Suppose that each $\phi_{n}$ is $\mu$-a.c. but the $\phi_{n}$ 's are not equi- $\mu$-a.c. Then there exists $\beta>0$ such that for each $n \geq 1$, we can find $A_{n} \in \mathscr{M}$ and $q_{n} \in \mathbb{N}$ such that

$$
\mu^{\#}\left(A_{n}\right)<2^{-n} \text { and } \phi_{q_{n}}\left(A_{n}\right)>\beta .
$$

Note that each $\phi_{q}$ is $\mu$-a.c., and so $\phi_{q}\left(A_{m}\right) \rightarrow 0$ as $m \rightarrow \infty$; therefore $q_{n} \rightarrow \infty$. So, passing to an appropriate subseqence of $\left(\phi_{q_{n}}\right)_{1}^{\infty}$, we may suppose that $q_{n}=n$ for each $n$.

Now let $T_{n}=\bigcup_{k \geq n} A_{k}$. If $n, p \in \mathbb{N}$, then

$$
\beta<\phi_{n}\left(A_{n}\right) \leq \phi_{n}\left(A_{n} \backslash T_{p}\right)+\phi_{n}\left(A_{n} \cap T_{p}\right)
$$

by (9) with $q_{n}=n$ and (SA), and $\mu^{\#}\left(A_{n} \cap T_{p}\right) \rightarrow 0$ as $p \rightarrow \infty$ by (9). Since $\phi_{n}$ is $\mu$-a.c., it follows that $\phi_{n}\left(A_{n} \backslash T_{p}\right)>\beta$ whenever $p$ is large enough $\left(p \geq p_{n}\right)$. Therefore, exactly as in the proof of Theorem 1, we can find natural numbers $p_{0}=1=n_{1}<p_{1}<n_{2}<p_{2}<\cdots$ such that

$$
\phi_{n_{k}}\left(B_{k}\right)>\beta \quad \forall k \geq 1,
$$

where $B_{k}=A_{p_{k-1}} \backslash T_{p_{k}}$. Notice that the $B_{k}$ 's are pairwise disjoint and $\mu^{\#}\left(B_{k}\right) \leq$ $\mu^{\#}\left(A_{p_{k-1}}\right) \rightarrow 0$ by (9). It follows from Lemma 5 that there exists $I \subset \mathbb{N}$ such that

$$
\phi_{n_{k}}\left(B_{I}\right) \geq \phi_{n_{k}}\left(B_{k}\right) / 2 \text { for infinitely many } k \text { 's, }
$$

where $B_{I}=\bigcup_{k \in I} B_{k}$. Combining $(*),(10)$, and (11), we get $\psi\left(B_{I}\right) \geq \beta / 2$. But it is clear that such a set $I$ can be chosen as a subset of any preassigned infinite set $J \subset \mathbb{N}$. So we can construct infinitely many, pairwise disjoint sets $B_{I}$ with $\psi\left(B_{I}\right) \geq \beta / 2$. Hence $\psi$ is nonvanishing.

If each $\phi_{n}$ is vanishing (or continuous) but the $\phi_{n}$ 's are not equi-vanishing (or not equi-continuous), it is easy to find a disjoint sequence $\left(B_{k}\right)_{1}^{\infty}$ in $\mathscr{M}$ and a subsequence $\left(\phi_{n_{k}}\right)_{1}^{\infty}$ of $\left(\phi_{n}\right)_{1}^{\infty}$ satisfying (10) for some $\beta>0$. So the above arguments with Lemma 5 replaced by Lemma 3 show that $\psi$ is nonvanishing, which completes the proof.

The following is a combination of the Vitali-Hahn-Saks Theorem and the Nikodym Convergence Theorem (see [1] or [3]).

Corollary 7. Let $\mu$ be a countably subadditive measuroid, let $X$ be a Banach space, and let $\left(\mu_{n}\right)_{1}^{\infty}$ be a sequence of finitely additive $X$-valued measures on $\mathscr{M}$ such that

$$
\nu(A):=\lim \mu_{n}(A)
$$

exists in norm for each $A \in \mathscr{M}$. Then $\nu$ is finitely additive. Moreover, letting $T=\{\nu\} \cup\left\{\mu_{n}\right\}_{1}^{\infty}$, we have:

(a) If each $\mu_{n}$ is $\mu$-a.c., then $T$ is equi- $\mu$-a.c. 
(b) If each $\mu_{n}$ is vanishing (i.e., strongly additive), then $T$ is equi-vanishing and bounded (relative to $\sigma \rightarrow \sigma^{\#}(\Omega)$ ).

(c) If each $\mu_{n}$ is continuous (i.e., countably additive), then $T$ is equicontinuous and bounded.

Proof. The first statement is trivial.

To prove (b) and (c), suppose that each $\mu_{n}$ is vanishing or continuous. Then $\mu_{n}^{\#}$ is vanishing by Lemma 2 . To force a contradiction, suppose that $\nu^{\#}$ is not vanishing. Then there exists a disjoint sequence $\left(B_{k}\right)_{1}^{\infty}$ in $\mathscr{M}$ and $\beta>0$ such that $\left\|\nu\left(B_{k}\right)\right\|>\beta$ for every $k$. Passing to a subsequence of $\left(\mu_{n}\right)_{1}^{\infty}$, we may suppose that

$$
\sum_{k=1}^{n}\left\|\nu\left(B_{k}\right)-\mu_{n}\left(B_{k}\right)\right\|<1 / n \quad \forall n \geq 1 .
$$

Recall that each $\mu_{n}^{\#}$ is vanishing. So, by applying Lemma 2(a), we may further suppose that

$$
\mu_{n}^{\#}\left(\bigcup_{k>n} B_{k}\right)<1 / n \quad \forall n \geq 1 .
$$

Now let $B=\bigcup_{k=1}^{\infty} B_{k}$. Then the finite additivity of $\mu_{n}$ ensures that

$$
\begin{aligned}
\left\|\nu(B)-\sum_{k=1}^{n} \nu\left(B_{k}\right)\right\| \leq & \left\|\nu(B)-\mu_{n}(B)\right\|+\left\|\mu_{n}(B)-\sum_{k=1}^{n} \mu_{n}\left(B_{k}\right)\right\| \\
& \quad+\sum_{k=1}^{n}\left\|\mu_{n}\left(B_{k}\right)-\nu\left(B_{k}\right)\right\| \\
\leq & \left\|\nu(B)-\mu_{n}(B)\right\|+\left\|\mu_{n}\left(\bigcup_{k>n} B_{k}\right)\right\|+1 / n \quad \text { by }(12) \\
\leq & \left\|\nu(B)-\mu_{n}(B)\right\|+2 / n \text { by }(13) .
\end{aligned}
$$

It follows from $(*)$ that $\left\|\nu(B)-\sum_{k=1}^{n} v\left(B_{k}\right)\right\| \rightarrow 0$ as $n \rightarrow \infty$, which is impossible since $\left\|\nu\left(B_{k}\right)\right\|>\beta$ for every $k$. This recuctio ad absurdum shows that if each $\mu_{n}$ is vanishing or continuous, then $\nu^{\#}$ is vanishing. Thus (b) and (c) follows from Theorem 6 combined with Theorem 5 .

Finally suppose that each $\mu_{n}$ is $\mu$-a.c. but the $\mu_{n}$ 's are not equi- $\mu$-a.c. Then the proof of Theorem 6 with $\psi(A)=\|\nu(A)\|$ yields pairwise disjoint sets $U_{k}\left(=B_{I_{k}}\right)$ in $\mathscr{M}$ such that $\sum_{1}^{\infty} \mu^{\#}\left(U_{k}\right)<\infty$ and $\left\|\nu\left(U_{k}\right)\right\| \geq \beta / 2$ for all $k$ 's and some $\beta>0$. But the arguments in the above two paragraphs show that this is impossible, which establishes (a).

The norm convergence assumption in Corollary 7 is too strong if the Banach space $X$ is good enough. Consider the following "fatness" condition on $X$ : given $C<\infty$, there exists $m \in \mathbb{N}$ such that $x_{1}, \ldots, x_{m} \in X$ and $\left\|x_{k}\right\| \geq 1$ for $1 \leq k \leq m$ implies $\left\|\sum_{k \in F} x_{k}\right\|>C$ for some $F \subset\{1,2, \ldots, m\}$. An easy application of Khintchine's Inequality shows that every $L_{p}$-space $(0<p<\infty)$ satisfies this condition, even though $\|\cdot\|_{p}$ is not a norm for $p<1$ (in general). 
Corollary 8. Suppose in Corollary 7 that $X$ satisfies the above fatness condition and, in place of norm convergence, that there exists a positive finite constant $\gamma$ such that

$$
\limsup _{n \rightarrow \infty}\left\|\mu_{n}(A)\right\| \leq \gamma \liminf _{n \rightarrow \infty}\left\|\mu_{n}(A)\right\|<\infty
$$

for each $A \in \mathscr{M}$. Then the conclusions (a), (b), and (c) of Corollary 7 obtain with $T=\left\{\mu_{n}\right\}_{1}^{\infty}$.

Proof. Let $\psi(A)$ denote the left-hand side of $(*)$. Suppose that $\psi$ is not vanishing. Then there exists a disjoint sequence $\left(B_{k}\right)_{1}^{\infty}$ in $\mathscr{M}$ and $\beta>0$ such that $\psi\left(B_{k}\right)>\beta$ for every $k$. Given $C<\infty$, let $m$ be the natural number furnished by the fatness condition. By our assumption on $\left(B_{k}\right)_{1}^{\infty}$ and $(*)$, there exists $n \geq 1$ such that $\beta<\gamma\left\|\mu_{n}\left(B_{j}\right)\right\|$ for $j=1, \ldots, m$. By our choice of $m$, we can find $F \subset\{1,2, \ldots, m\}$ such that

$$
\left\|\mu_{n}\left(\bigcup_{j \in F} B_{j}\right)\right\|=\left\|\sum_{j \in F} \mu_{n}\left(B_{j}\right)\right\| \geq C \beta / \gamma
$$

As $C<\infty$ is arbitrary, it follows that the $\mu_{n}$ 's are not uniformly bounded. This fact, combined with $(*)$ and Theorem 4 , shows that if each $\mu_{n}$ is vanishing, then $\psi$ is vanishing. Hence (b) and (c) of Corollary 7 with $T=\left\{\mu_{n}\right\}_{1}^{\infty}$ follows from Theorem 6 .

For (a), we argue as follows. Suppose that each $\mu_{n}$ is $\mu$-a.c. but the $\mu_{n}$ 's are not equi- $\mu$-a.c. Then the above $\left(B_{k}\right)_{1}^{\infty}$ can be chosen to satisfy $\sum_{1}^{\infty} \mu^{\#}\left(B_{k}\right)<\infty$ (see the last paragraph of the last proof). But $X$ is supposed to satisfy the fatness condition, so $(*)$ ensures that there exists a disjoint sequence $\left(F_{k}\right)_{1}^{\infty}$ of finite subsets of $\mathbb{N}$ and a subsequence $\left(\mu_{n_{k}}\right)_{1}^{\infty}$ of $\left(\mu_{n}\right)_{1}^{\infty}$ such that $\left\|\mu_{n_{k}}\left(\bigcup_{j \in F_{k}} B_{j}\right)\right\|>k$ for each $k \geq 1$. Since each $\mu_{n_{k}}$ is $\mu$-a.c. and $\mu^{\#}\left(\bigcup_{j \in F_{k}} B_{j}\right) \rightarrow 0$ as $k \rightarrow \infty$, it follows from Lemma 5 that there exists $I \subset \mathbb{N}$ such that $\left\|\mu_{n_{k}}\left(\bigcup_{j \in I} B_{j}\right)\right\|>k / 2$ for infinitely many $k$ 's. Plainly this contradicts $(*)$, which establishes $(a)$.

Remarks. (i) Let $\left(\phi_{n}\right)_{1}^{\infty}$ be a sequence of vanishing measuroids on $2^{\mathbb{N}}$ such that $\phi_{n}(A) \rightarrow 0$ for each $A \subset \mathbb{N}$. Then

$$
\sup \left\{\phi_{n}^{\#}(F): F \subset \mathbb{N} \text { is finite }\right\} \rightarrow 0
$$

as $n \rightarrow \infty$ (Philips' Lemma). This is an almost immediate consequence of Lemma 3.

(ii) In Theorem 6 and Corollaries 7 and 8 , it is not difficult to deduce both the "vanishing" and "continuous" cases from the " $\mu$-a.c." case.

(iii) Let $f$ be a strictly increasing continuous unbounded function on $[0, \infty)$ with $f(0)=0$, and let $f(\infty)=\infty$. In the definition of a measuroid, replace (SA) and (SB) by

$$
\begin{aligned}
& \phi(A \cup B) \leq f(\phi(A))+f(\phi(B)), \\
& \phi(A) \leq f(\phi(A \cup B))+f(\phi(B)),
\end{aligned}
$$

respectively. If $f$ is fixed, all of our results about measuroids remain valid even in this generalized setting, except that the inequality in Lemmas 3 and 5 must now be replaced by

$$
f\left(\phi_{n}\left(B_{I}\right)\right)+(f \circ f)\left(\phi_{n}\left(B_{I}\right)\right) \geq \phi_{n}\left(B_{n}\right) .
$$


This generalization appears to be necessary when we study maximal operators of various types.

(iv) The middle term of the inequalities in Corollary 8 may be replaced by $f\left(\liminf _{n \rightarrow \infty}\left\|\mu_{n}(A)\right\|\right)$, where $f$ is as in the above remark.

\section{REFERENCES}

1. P. Antosik and C. Swartz, Matrix methods in analysis, Lecture Notes in Math. vol. 1113, Springer-Verlag, Berlin, Heidelberg, New York, and Tokyo, 1985.

2. C. Bessaga and A. Pelczynski, On bases and unconditional convergence of series in Banach spaces, Studia Math. 17 (1958), 151-164.

3. J. Diestel, Sequences and series in Banach spaces, Grad. Texts in Math., no. 92, SpringerVerlag, Berlin, Heidelberg, New York, and Tokyo, 1984.

4. J. Diestel and B. Faires, On vector measures, Trans. Amer. Math. Soc. 198 (1974), 253-271.

Department of Mathematics, Kansas State University, Manhattan, Kansas 66506 